\title{
The influence of substituents on gelation and stacking order of oligoaramid - based supramolecular networks $\dagger$
}

\author{
Susanne Drechsler, ${ }^{\mathrm{a}}$ Sandor Balog, $\mathbb{D D}^{\mathrm{b}}$ Andreas F. M. Kilbinger (DD *a and \\ Tommaso Casalini* ${ }^{* d}$
}

\begin{abstract}
Self-assembly has proven to be a powerful tool for functional, smart materials such as hydrogels derived from low molecular weight compounds. However, the targeted design of functional gelators remains difficult. Here, we present a set of four $Y$-shaped aromatic amide tetramers with varying functionalities able to undergo different non-covalent interactions. These compounds were explored towards their self-assembly behavior and hydrogel formation by experimental methods such as UV-vis spectroscopy, rheology, small angle X-ray scattering (SAXS), scanning/transmission electron, and atomic force microscopy. Additionally, we investigated the main mechanisms behind oligomer aggregation and the structure of the resulting supramolecular chains through full atomistic molecular dynamics simulations.
\end{abstract}

\section{Introduction}

Self-assembly provides a multitude of possibilities for the design of functional and smart materials such as supramolecular polymers ${ }^{1,2}$ host-guest interactions ${ }^{3}$ for catalysis and sensors, ${ }^{4}$ biomedical compounds for drug delivery systems ${ }^{5}$ or soft materials such as hydrogels derived from low molecular weight compounds. ${ }^{6,7}$ All these systems are based on dynamic noncovalent interactions responsible for the unique, often stimuli responsive materials. Very promising candidates for the use in supramolecular chemistry-based systems are rigid molecules. The interest in these shape-persistent compounds arises due to the fact that they can adopt only very defined conformations, which later on lead to precise and sometimes predictable supramolecular nanostructures. One very interesting class within the field of rigid molecules are aromatic oligoamides (oligoaramids).

${ }^{a}$ Department of Chemistry, University of Fribourg, Chemin du Musée 9, CH-1700 Fribourg, Switzerland. E-mail: andreas.kilbinger@unifr.ch

${ }^{b}$ Adolphe Merkle Institute, University of Fribourg, Chemin des Verdiers 4, CH-1700 Fribourg, Switzerland

${ }^{c}$ Institute for Chemical and Bioengineering, Department of Chemistry and Applied Biosciences, ETH Zurich, 8093 Zurich, Switzerland.

E-mail: tommaso.casalini@chem.ethz.ch

${ }^{a}$ Institute of Mechanical Engineering and Material Technology,

Department of Innovative Technologies, SUPSI, 6928 Manno, Switzerland

$\dagger$ Electronic supplementary information (ESI) available: Experimental procedures and ${ }^{1} \mathrm{H}$ NMR, ${ }^{13} \mathrm{C}$ NMR peak reports and HR-mass for all compounds, photographs gelation experiments under different conditions, additional rheology data, AFM/ SEM/ TEM images, calculated molecular dimensions, polarized-light micrographs computational parameters and detailed molecular dynamic simulation protocols. See DOI: 10.1039/c9sm00148d
Targeted substitution on the aromatic ring leads to a spatially well-defined positioning of functionalities serving as features for molecular organization. ${ }^{8}$ Oligoaramids have been used for instance to mimic biological macromolecules. ${ }^{9}$ As oligomers can be synthesized so that they can adopt only few defined conformations, they have been extensively used in the field of foldamers. ${ }^{10}$ Suitable building blocks for oligoaramids, which allow for a modular synthesis based on well-explored peptide chemistry, are aminobenzoic acid (ABA) derivatives. Oligomers or polymers from these monomeric units have the ability to interact via hydrogen bonding between their amide bonds or $\pi-\pi$ stacking between the aromatic rings. ${ }^{11}$ These interactions are a prerequisite for the formation of supramolecular structures. However, rigid molecules often suffer from low solubility, limiting their otherwise promising utilization for materials with intriguing properties. Research focuses preferentially on organo-solubility of these compounds.

Since aramids are well established to function as compounds for nano-architectures and supramolecular chemistry based soft materials ${ }^{12-15}$ we anticipated that they could be good candidates for low molecular weight hydrogelators. However, the targeted design of functional gelators remains challenging and the synthesis of amphiphilic aramide oligomers has only been addressed by few groups. ${ }^{16-18}$ Therefore, we present herein the synthesis of amphiphilic aromatic amide oligomers and the investigation of their aqueous gelation behavior by rhelology, scattering methods (SAXS) and imaging techniques such as AFM and SEM. Insight into the molecular aggregation mechanism was gained using full atomistic molecular dynamics simulations. 


\section{Results and discussion}

Gelator synthesis

Low molecular weight gelators (LMWG) for hydrogels are based on a delicate balance between hydrophobic and hydrophilic groups. We believed that aromatic amide oligomers could be excellent candidates for the design of LMWG as they can be constructed sequentially from hydrophilic and hydrophobic amino acid building blocks.

For this purpose, a set of aramid oligomers was synthesized, differing in the arrangement of hydrophilic and hydrophobic building blocks. A 4-aminosalicylic acid derivative carrying a tertiary amine in the side chain (Fig. 1, indicated by light blue building blocks) was used as the water-soluble building block. As hydrophobic building blocks, we used either 4-nitrobenzoic acid, 4-aminobenzoic acid or methyl 4-aminobenzoate.

Seven different oligomers were synthesized from these building blocks as shown in Fig. 1. The oligomers differed in shape and in $\mathrm{C}$ - and $\mathrm{N}$-terminal functional groups (carboxylic acids/esters $v s$. amines and nitro groups).

Four branched tetramers were synthesized, carrying an $\mathrm{N}$-terminal nitro group and two C-terminal methyl esters ( $\mathrm{NO}_{2} \mathrm{COOMe}-\mathrm{Y}$ ), an $\mathrm{N}$-terminal nitro group and two C-terminal carboxylic acids ( $\left.\mathbf{N O}_{2} \mathbf{C O O H}-\mathbf{Y}\right)$, an N-terminal amine and two C-terminal methyl esters ( $\left.\mathbf{N H}_{\mathbf{2}} \mathbf{C O O M e - Y}\right)$ or an N-terminal amine and two C-terminal carboxylic acids $\left(\mathbf{N H}_{2} \mathbf{C O O H}-\mathbf{Y}\right)$. Each branched tetramer consisted of two hydrophilic building blocks (the C-terminal amino acids) and two hydrophobic building blocks (the N-terminal and branching amino acid).

Gelation experiments were carried out at $3 \mathrm{wt} \%$ of the branched tetramer in $0.01 \mathrm{M} \mathrm{NaCl}_{\mathrm{aq}}$ (reference conditions, under these conditions, gelation was found to be stronger than in pure water while still giving clear gels, see Fig. S1, ESI $\dagger$ ).

All branched (Y-shaped) gelators except $\mathbf{N H}_{2} \mathbf{C O O M e}-\mathbf{Y}$ showed the formation of gels in the inverted-vial-test (see photo in Fig. 1, center).

To gain further insight into the structural requirements for such aramid hydrogelators, we synthesized a symmetric Y-shaped hydrophilic oligomer (Fig. 1, sym(COOMe) $)_{3}-\mathrm{Y}$, bottom) and two amphiphilic branched oligomers differing in the length of the hydrophobic rigid rod-like part (Fig. 1, short $\mathbf{N O}_{2}$ COOMe-Y, $\mathrm{NO}_{2} \mathrm{ArCOOH}-\mathrm{Y}$, bottom). It was found, that neither $\mathbf{S y m}(\mathrm{COOMe})_{3}-\mathbf{Y}$ nor short $\mathrm{NO}_{2} \mathrm{COOMe}-\mathrm{Y}$ were able to form gels despite their good water solubility. This confirms that the rigid hydrophobic rods within the oligomers are necessary for self-assembly. Extension of the hydrophobic rigid rod by one phenyl unit as is the case in $\mathbf{N O}_{2}$ ArCOOH-Y leads to turbid aqueous solutions with weak gelation tendencies.

Therefore, only the gelation behavior of the branched tetramers (Fig. 1, center) was further investigated.

\section{Solvent dependent aggregation}

When ${ }^{1} \mathrm{H}$-NMR experiments of the four branched tetramers ( $\mathrm{NO}_{2} \mathrm{COOMe}-\mathrm{Y}, \mathbf{N O}_{2} \mathrm{COOH}-\mathbf{Y}, \mathbf{N H}_{2} \mathbf{C O O M e}-\mathbf{Y}$ and $\mathbf{N H}_{2} \mathrm{COOH}-\mathrm{Y}$ ) were conducted in $\mathrm{D}_{2} \mathrm{O}$, we detected broadening and an up-field shift of the aromatic peaks indicative of aromatic stacking. ${ }^{19}$
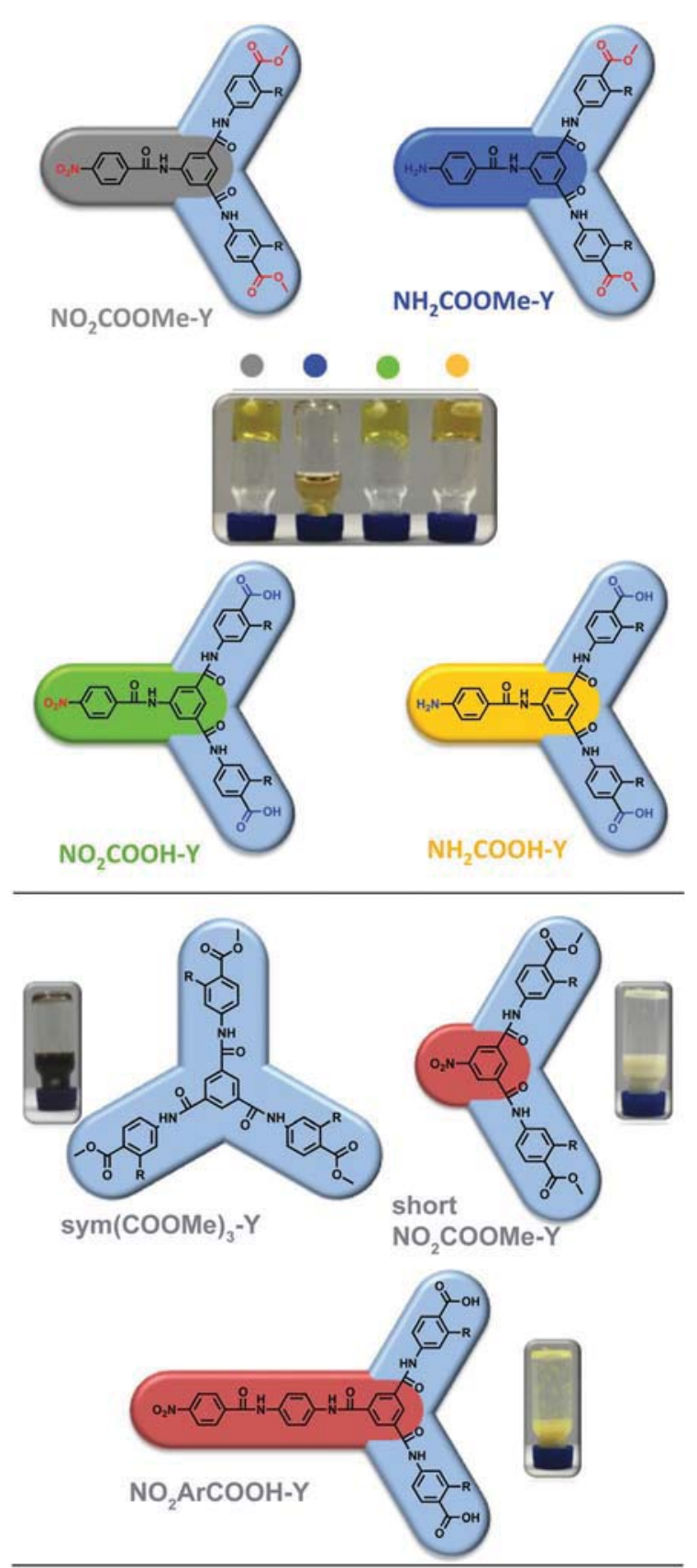

For all compounds: $\quad \mathbf{R}=. \mathrm{O}^{\mathrm{N}}$

Fig. 1 Structures of potential gelators and corresponding photographs of the inverted-vial-test under reference conditions (3 wt\% in $0.01 \mathrm{M} \mathrm{NaCl}_{\mathrm{aq}}$.).

In DMSO- $d_{6}$ on the other hand well-defined sharp peaks were observed indicating a molecularly dissolved state $\left({ }^{1} \mathrm{H}-\mathrm{NMR}\right.$ spectra in $\mathrm{D}_{2} \mathrm{O}$ and DMSO see ESI, $\dagger$ Fig. S2). This behavior was further supported by UV-vis measurements in DMSO $(c=10 \mu \mathrm{M})$ and aqueous $(c=10 \mu \mathrm{M})$ solutions. The vibronic fine structure of the absorption peaks observed in DMSO solution was lost due to peak broadening when solutions in water were measured (see ESI $\dagger$ Fig. S5), indicative of aromatic stacking. ${ }^{19,20}$ All four compounds show signs of aggregation in ${ }^{1} \mathrm{H}-\mathrm{NMR}$ and UV/vis spectroscopy. However, no gelation was observed for 
$\mathbf{N H}_{2}$ COOMe-Y. This indicates that the length scales on which aggregation occurs might be significantly different for $\mathbf{N H}_{2}$ COOMe-Y in comparison to the other three compounds. This assumption was subsequently confirmed by small angle X-ray scattering (vide infra).

\section{Mechanical characterization by rheology}

In order to quantify gel strengths rheology experiments were carried out on gels of all branched tetramers. Measurements were carried out by dissolving all branched tetramer gelators in aqueous $\mathrm{NaCl}$ solution $(0.01 \mathrm{M})$. However, $\mathbf{N H}_{2} \mathbf{C O O M e}-\mathbf{Y}$ (Fig. 2, blue) did not form a gel under these conditions and was omitted from the measurements.

At first, the linear viscoelastic range (LVE) was identified by carrying out a strain sweep experiment at a constant frequency of $1 \mathrm{~Hz}$ (see ESI, $\dagger$ Fig. S3). It was found, that all gel samples showed an LVE behaviour at a strain of 1\% under the tested conditions. Subsequently, frequency sweep experiments were performed at a constant strain of $1 \%$ (see Fig. 3). The observed $G^{\prime}$ and $G^{\prime \prime}$ values (see ESI, $\dagger$ Table S1) give information to what extent the sample behaves like an elastic solid $\left(G^{\prime} / G^{\prime \prime}>1\right)$ or a viscous liquid $\left(G^{\prime} / G^{\prime \prime}<1\right)$. $\mathbf{N O}_{2} \mathbf{C O O M e - Y}$ (Fig. 2, grey) displayed the highest storage modulus (800 $\mathrm{Pa}$ ) of all gelators investigated (see Fig. 3). Compounds $\mathbf{N O}_{2} \mathbf{C O O H}-\mathbf{Y}$ and $\mathbf{N H}_{2} \mathbf{C O O H}-\mathbf{Y}$ can also be described as gels, but exhibiting a lower $G^{\prime}$ of $290 \mathrm{~Pa}$ and $110 \mathrm{~Pa}$, respectively.

Plotting the $G^{\prime} / G^{\prime \prime}$ ratio $v s$. gelator concentration showed that all three gelators form stable gels even at lower concentrations (1\% for $\mathbf{N O}_{2} \mathbf{C O O M e - Y , ~} 2 \%$ for $\mathbf{N O}_{2} \mathbf{C O O H}-\mathbf{Y}$ and $\mathbf{N H}_{2} \mathbf{C O O H}-\mathbf{Y}$, see Fig. 3).

\section{Small angle X-ray scattering}

In order to obtain information about the length scales at which the self-assembly processes occurred in the gels investigated, we performed small-angle X-ray scattering (SAXS) experiments

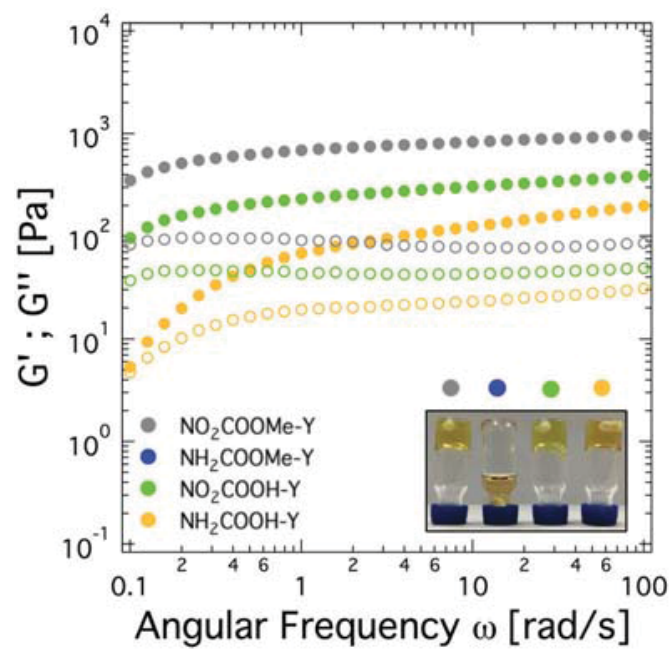

Fig. 2 Rheological data derived from oscillatory frequency sweep experiments at constant strain of $1 \%$ (within LVE) for gels prepared from $0.01 \mathrm{M}$ $\mathrm{NaCl}_{\text {aq. }}$. All gels with a final gelator concentration of $c=37.5 \mathrm{mM}$ (ca. $3 \mathrm{wt} \%$ ). Filled-in circles represent $G^{\prime}$ and holow circled represent $G^{\prime \prime}$.

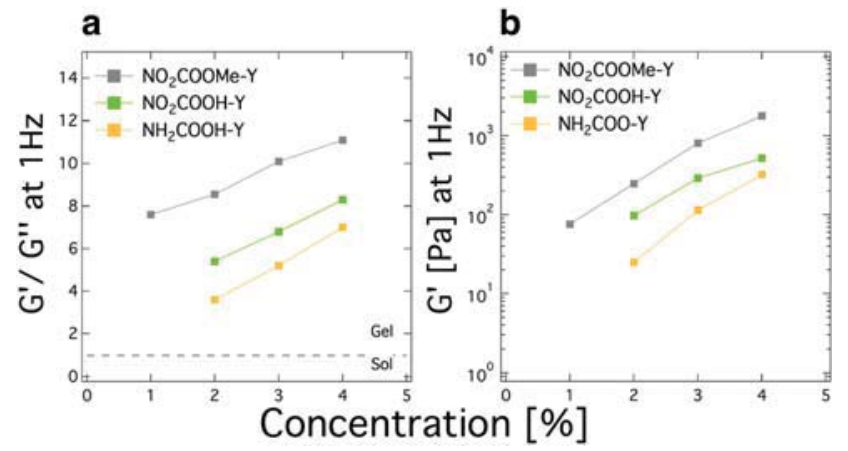

Fig. 3 Concentration dependence of gels from $10 \mathrm{mM} \mathrm{NaCl}$ aq. solutions: (a) $G^{\prime} / G^{\prime \prime}$ ratio $\left(G^{\prime} / G^{\prime \prime}>1\right.$ criteria for elastic solid-like gels) and (b) $G^{\prime}$ representing gel strength. Values reported from frequency sweep experiments at $1 \mathrm{~Hz}$ and a constant strain of $1 \%$ and $25{ }^{\circ} \mathrm{C}$ (within the linear viscoelastic range).

as shown in Fig. 4 . Reference conditions ( $3 \mathrm{wt} \%$ in $0.01 \mathrm{M} \mathrm{NaCl}_{\mathrm{aq}}$ ) were used in order to compare results to other analysis methods such as microscopic imaging (see below). SAXS spectra under these conditions show clearly the differences between the gelating compounds $\mathrm{NO}_{2} \mathrm{COOMe}-\mathrm{Y}, \mathrm{NO}_{2} \mathrm{COOH}-\mathrm{Y}, \mathrm{NH}_{2} \mathrm{COOH}-\mathrm{Y}$ and $\mathbf{N H}_{2} \mathbf{C O O M e}-Y$, from which only solutions were obtained.

SASView $^{21}$ was used to fit the SAXS data to a cylindrical model (see Table S3 for parameters used, ESI $\dagger$ ). Good-quality fits were obtained for the three gelating compounds. The radii of the models were found to be in the size range of the molecular dimensions of the respective compounds (around $2 \mathrm{~nm}$, see Fig. 4).

In contrast, for $\mathbf{N H}_{2} \mathbf{C O O M e}-\mathbf{Y}$ only half of the diameter was obtained $(d=1.04 \mathrm{~nm}$, Fig. $4 \mathrm{~b})$. This strongly suggests that the aggregation in this case is of a different nature and may not be
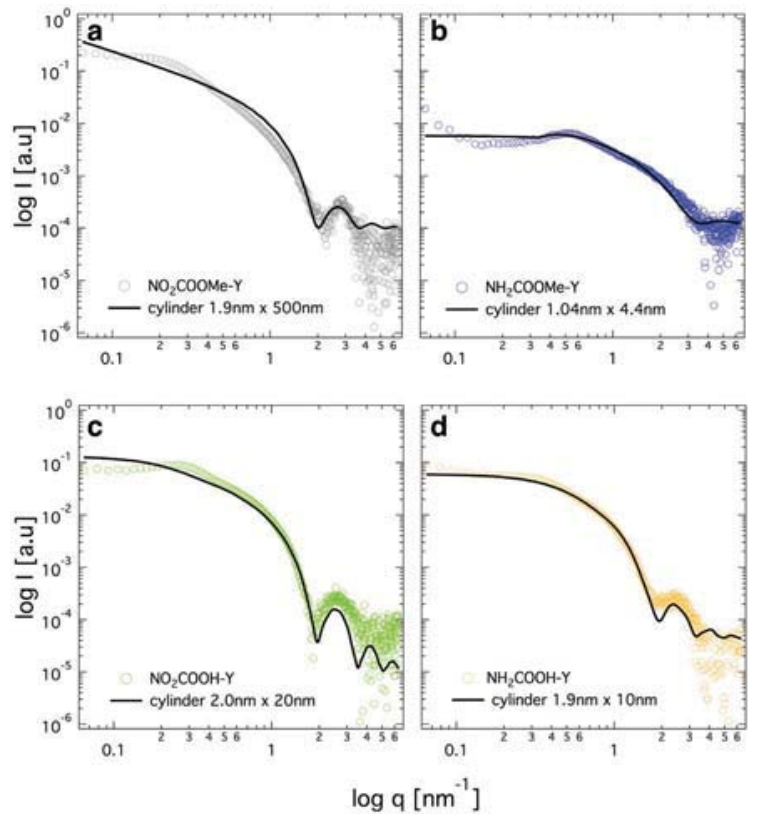

Fig. 4 SAXS fit with cylindrical model done with SasView. ${ }^{21}$ SAXS data obtained from 3 wt\% gelator in $0.01 \mathrm{M} \mathrm{NaCl}_{\mathrm{aq}}$ of (a) $\mathrm{NO}_{2} \mathrm{COOMe}-\mathrm{Y}$, (b) $\mathrm{NH}_{2} \mathrm{COOMe}-\mathrm{Y}$, (c) $\mathrm{NO}_{2} \mathrm{COOH}-\mathrm{Y}$ and (d) $\mathrm{NH}_{2} \mathrm{COOH}-\mathrm{Y}$. 
describable by the cylindrical model. The length dimensions of the cylinder seemed to be the longest for $\mathbf{N O}_{2} \mathbf{C O O M e}-\mathbf{Y}$ (around $500 \mathrm{~nm}$, Fig. 4a). However, it must be noted that with the SAXS setup used only data up to length ranges of $80 \mathrm{~nm}$ can be obtained. Therefore, the length of $\mathbf{N O}_{2} \mathbf{C O O M e - Y}$ must be seen as an approximation. Nonetheless, this observation is in agreement with rheology, were the highest gel strength (possibly the longest entangled fibers) was determined for this compound.

For $\mathbf{N O}_{2} \mathbf{C O O H}-\mathbf{Y}$ and $\mathbf{N H}_{2} \mathbf{C O O H}-\mathbf{Y}$, the length dimensions were found to be in a medium range of $20 \mathrm{~nm}$ (Fig. 4c) and $10 \mathrm{~nm}$ (Fig. 4d), respectively. As already mentioned, applying the same cylindrical model for $\mathbf{N H}_{\mathbf{2}} \mathbf{C O O M e}-\mathbf{Y}$ delivered no satisfying results, unless very short length dimensions were used $(1.04 \mathrm{~nm} \times 4.4 \mathrm{~nm}$, Fig. $4 \mathrm{~b})$.

\section{Microscopic investigation of assembled structures}

In order to investigate the aggregation behavior leading to macroscopic hydrogels, different microscopic experiments were performed: scanning electron microscopy (SEM) of freeze-dried xerogels, transmission electron microscopy (TEM) and atomic force microscopy (AFM) (see Fig. 5). For SEM experiments xerogels (under reference conditions) were prepared, which are believed to maintain important microscopic features of the gel. SEM revealed highly porous morphologies for all four compounds. For the non-gelating $\mathbf{N H}_{\mathbf{2}} \mathbf{C O O M e - Y}$ these morphologies were found to be slightly different (Fig. 5b). There, a finer sponge-like, interwoven network, was observed which is rather subtle compared to the other gelating compounds (Fig. 5a, c and d). These morphologies suggest that even $\mathbf{N H}_{2}$ COOMe-Y forms supra-molecular networks. However, they might not be strong enough to generate a macroscopic gel able
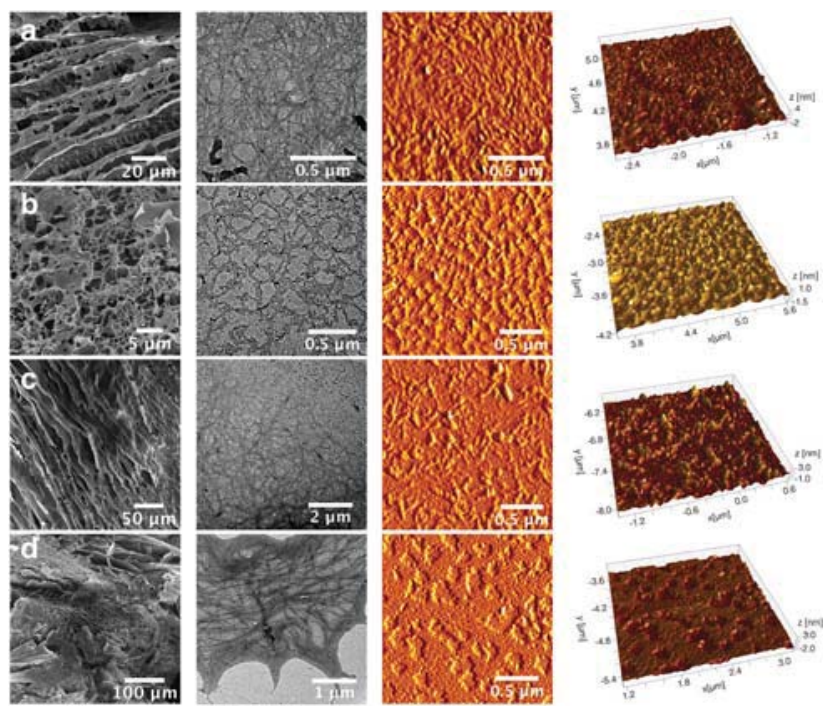

Fig. 5 From left to right: SEM photographs of xerogels (freeze-dried from $3 \mathrm{wt} \%$ gels in $10 \mathrm{mM} \mathrm{NaCl}$ aqueous solutions), TEM micrographs (2.5 mg mL $\mathrm{mb}^{-1}$ in $10 \mathrm{mM} \mathrm{NaCl}$ aqueous solutions) and AFM micrographs with $3 \mathrm{D}$ topography $\left(2.5 \times 10^{-2} \mathrm{mg} \mathrm{mL}^{-1}\right.$ in $10 \times 10^{-2} \mathrm{mM} \mathrm{NaCl}$ aqueous solutions) of (a) $\mathrm{NO}_{2} \mathrm{COOMe}-\mathrm{Y}$, (b) $\mathrm{NH}_{2} \mathrm{COOMe}-\mathrm{Y}$, (c) $\mathrm{NO}_{2} \mathrm{COOH}-\mathrm{Y}$ and (d) $\mathrm{NH}_{2} \mathrm{COOH}-\mathrm{Y}$. (See also Fig. S6-S10 in the ESI $\uparrow$ for additional TEM and AFM images). to support its own weight. Interestingly, the structures of the other three xerogels (Fig. 5a, c and d) consisted of sheet-like morphologies rather than fibers. Such sheets can currently not be correlated with a model of molecular self-assembly (see below).

To get a better insight into the aggregation behavior on sub-micrometer dimensions, TEM imaging was performed. Solutions of potential gelators $\left(2.5 \mathrm{mg} \mathrm{mL}^{-1}\right)$ were drop-cast on a carbon coated copper-grid and left drying overnight protected from dust. Here, the formation of an entangled fiber-network (see Fig. 5), which are typical for gels ${ }^{22-26}$ were observed for all four samples. ${ }^{22-26}$

The fibrillar structures were not found to be uniform. Their width ranged from 10-60 nm with several hundred $\mathrm{nm}$ in length for $\mathrm{NO}_{2} \mathrm{COOMe}-\mathrm{Y}, \mathrm{NO}_{2} \mathbf{C O O H}-\mathrm{Y}$ and $\mathbf{N H}_{2} \mathbf{C O O H}-Y$. These dimensions suggest, that these fibers are aggregates of the primary self-assembled structures. For $\mathbf{N H}_{2} \mathbf{C O O M e}-\mathbf{Y}$ the observed fibers did not exhibit such a broad range in length but the formation of a less entangled network, which also supports the macroscopic observation that no gels are formed.

AFM imaging of gels deposited on mica sheets was carried out as well. It was found that in case of the nitro group carrying tetramers $\left(\mathbf{N O}_{2} \mathbf{C O O M e - Y}\right.$ and $\left.\mathbf{N O}_{2} \mathbf{C O O H}-\mathbf{Y}\right)$ elongated structures were formed, that merged into a layer, which still exhibited a fibrous relief (see Fig. 5, 3D topology images). In case of amino group carrying compounds $\mathbf{N H}_{2} \mathbf{C O O M e - Y}$ and $\mathbf{N H}_{2} \mathbf{C O O H}-\mathbf{Y}$ these structures exhibited a more spherical character. The differences in morphology observed in TEM and AFM images are most likely due to the different substrates the samples were deposited on. For TEM measurements hydrophobic carboncoated copper grids were used while AFM measurements were performed on hydrophilic mica sheets.

\section{Summary of experimental data}

The experimental data described above clearly show that $\mathrm{NO}_{2} \mathbf{C O O M e}-\mathbf{Y}$ forms the strongest hydrogels, followed by $\mathbf{N O}_{2} \mathbf{C O O H}-\mathrm{Y}$ and $\mathbf{N H}_{2} \mathbf{C O O H}-\mathbf{Y}$ which are of similar strength but significantly weaker than $\mathbf{N O}_{2} \mathbf{C O O H}-Y$. For $\mathbf{N H}_{2} \mathbf{C O O M e - Y}$ no macroscopic gel formation was observed, but SEM images of xerogels formed by freeze drying of $\mathbf{N H}_{2} \mathbf{C O O M e}-\mathbf{Y}$ gels as well as TEM images indicate that even here, self-assembled structures are formed in aqueous solution.

Except for the functional group located at the termini of the three-arm stars, the four branched tetramers resemble each other geometrically (Fig. 1). Clearly, such small changes on the molecular level result in large effects macroscopically. Aromatic amide oligomers typically form aggregates via hydrogen bonding. However, in an aqueous environment this is unlikely to be the driving force for the observed self-assembly. We hypothesized that rigid three-arm star-shaped objects consisting mainly of aromatic structures are likely to form eclipsed aggregates in which the individual arms of the star-shaped objects maximize the attractive interactions with each other.

For three-arm star-like objects of $C_{2 \mathrm{v}}$ symmetry such as our branched tetramers there are two energetically distinguishable eclipsed dimers conceivable. The first dimer could be formed 
by stacking two stars on top of each other such that all like end groups are in contact with each other. The other possible arrangement would result in one like and two unlike end group contacts.

To be able to evaluate the most likely aggregate arrangement one would have to take into consideration the polarity, charge and solvation of the end groups in all possible arrangements. We therefore decided to carry out molecular dynamics (MD) simulations to establish a molecular level aggregation model.

\section{Molecular modeling of oligomer assembly}

The above experimental investigations have clearly shown that minute chemical modifications on the oligomer structure can have dramatic effects as far as self-assembly and macroscopic, i.e. gelation phenomena are concerned. An a priori detailed molecular image of the aggregation process (and many selfassembly processes for that matter) is, however, speculative at best.

The time and length scales accessible to atomistic MD do not allow the detailed study of the gelation dynamics, which will, therefore, not be considered from a computational point of view. It is, however, suitable to investigate the early aggregation stage, where monomers assemble in fibrous structures, in terms of interaction energies and structural parameters. A detailed overview of the simulation protocols is reported in the ESI. $\dagger$

First of all, each monomer was placed in a box filled with TIP3P explicit water molecules and $400 \mathrm{~ns}$ molecular dynamics simulations in $N P T$ ensemble at $300 \mathrm{~K}$ and 1 atm were performed. In order to obtain preliminary insights, solvation free energy values $\Delta G_{\text {solv }}$ were computed for the last $100 \mathrm{~ns}$ using the Generalized Born approach, ${ }^{27-29}$ averaging over multiple configurations; values are reported in Table 1.

In parallel, we investigated the main phenomena behind the self-assembly by studying the formation of dimers by means of Well - Tempered Metadynamics simulations (WTMD). ${ }^{30}$ Briefly, metadynamics simulations allow to obtain the free energy of the system of interest as a function of a few relevant conformational parameters (usually referred to as collective variables). In this framework, the hydrophobic contacts between the centers of mass of the aromatic rings (representative of $\pi-\pi$ interactions) and the Debye-Hückel energy (representative of electrostatic interactions) were chosen as suitable collective variables $(\mathrm{CV})$. It is important to note that a high value for hydrophobic contacts implies a more stable and ordered stacking between the rings.
Two monomers of each compound were placed in a box filled with TIP3P water molecules; after equilibration, $320 \mathrm{~ns}$ metadynamics simulations were carried out. The convergence was checked by computing the dimerization free energy as a function of simulation time (see ESI $\dagger$ ). Dimerization free energy values $\Delta F_{\text {dim }}$ are reported in Table 1 ; they were computed using the last $40 \mathrm{~ns}$ of WTMD simulations, where convergence had been achieved (see ESI $\dagger$ ). Free energy surfaces (FES) as a function of both $\mathrm{CV}$ are reported in $\mathrm{ESI} \dagger$ section.

To analyze in more detail the mechanisms behind dimerization, we selected a representative minimum energy structure for each dimer from the WTMD simulations, shown in Fig. 6, and we performed additional $100 \mathrm{~ns}$ MD simulations in NPT ensemble at $300 \mathrm{~K}$ and $1 \mathrm{~atm}$ with explicit TIP3P water molecules. Hydrophobic contacts and Debye-Hückel energies were computed from molecular trajectories; the average values (listed in Table 1) are consistent with the minimum energy one highlighted by WTMD outcomes. They also showed a fast convergence as a function of simulation time (see ESI $\dagger$ ) indicating that dimer structures remained stable during the simulation.

As can be seen from Table 1, dimerization free energy does not show a clear trend with solubility (as could be expected) but seems to be more related to the specific interactions between the monomers. Focusing now on tetramers, data from molecular simulations suggest that dimerization free energy is mainly due to hydrophobic contacts, since they exhibit the same trend, rather than the intrinsic solubility.

The aggregation of $\operatorname{sym}(\mathrm{COOMe})_{3}-\mathbf{Y}, \mathbf{N O}_{2} \mathbf{C O O M e - Y}$ and $\mathbf{N H}_{\mathbf{2}} \mathbf{C O O M e}-\mathbf{Y}$ led to the most energetically favorable dimers, resulting from the compromise between hydrophobic effects and electrostatic repulsion (positive Debye-Hückel energy), by virtue of a net charge equal to $+3 e$ for $\operatorname{sym}(\mathrm{COOMe})_{3}-\mathrm{Y}$ and $+2 e$ for $\mathbf{N O}_{2} \mathbf{C O O M e}-\mathbf{Y}$ and $\mathbf{N H}_{\mathbf{2}} \mathbf{C O O M e}-\mathbf{Y}$ due to the protonation of the tertiary amines in the side chains in neutral water. As shown in Fig. 6, representative dimer configurations confirm our initial assumption that an eclipsed orientation is favored. The repulsion is dampened by the formation of hydrogen bonds with the carbonyl oxygen of the methyl ester group (vide infra).

Focusing on $\operatorname{sym}(\mathbf{C O O M e})_{3}-\mathbf{Y}$ monomer, Debye-Hückel energy of is largely positive, suggesting that the strong electrostatic repulsion can still hinder the formation of a gel by disfavoring the attainment of large aggregates able to build a supramolecular network.

On the other hand, the electrostatic repulsion of $\mathrm{NO}_{2} \mathbf{C O O M e}-\mathbf{Y}$ and $\mathbf{N H}_{2} \mathbf{C O O M e}-\mathbf{Y}$ monomers is alleviated by introducing a nitro

Table 1 Solvation free energy of a single monomer, dimerization free energy from WTMD simulations, hydrophobic contacts and Debye-Hückel energy from MD simulation of dimer for each considered compound. Values are expressed as average \pm standard deviation

\begin{tabular}{|c|c|c|c|c|c|}
\hline Compound & $\Delta G_{\text {solv }}\left[\mathrm{kcal} \mathrm{mol}^{-1}\right]$ & & $\Delta F_{\text {dim }}\left[\mathrm{kcal} \mathrm{mol}^{-1}\right]$ & Hydrophobic contacts [-] & Debye-Hückel energy $\left[\mathrm{kJ} \mathrm{mol}^{-1}\right]$ \\
\hline $\operatorname{sym}(\mathrm{COOMe})_{3}-\mathrm{Y}$ & $-226.57 \pm 9.80$ & & $-10.13 \pm 0.13$ & $3.07 \pm 0.24$ & $7.50 \pm 0.66$ \\
\hline $\mathrm{NO}_{2} \mathrm{COOMe}-\mathrm{Y}$ & $-160.58 \pm 9.89$ & & $-9.61 \pm 0.21$ & $3.14 \pm 0.25$ & $2.56 \pm 0.67$ \\
\hline $\mathrm{NH}_{2} \mathrm{COOMe}-\mathrm{Y}$ & $-154.56 \pm 9.70$ & $\frac{7}{6}$ & $-7.49 \pm 0.13$ & $2.92 \pm 0.29$ & $2.49 \pm 0.66$ \\
\hline Short $\mathrm{NO}_{2} \mathrm{COOMe}-\mathrm{Y}$ & $-145.66 \pm 8.82$ & 总 & $-3.66 \pm 0.04$ & $2.17 \pm 0.27$ & $3.38 \pm 0.52$ \\
\hline $\mathrm{NO}_{2} \mathrm{COOH}-\mathrm{Y}$ & $-130.58 \pm 13.71$ & $\frac{2}{2}$ & $-6.94 \pm 0.32$ & $2.58 \pm 0.38$ & $-1.21 \pm 0.77$ \\
\hline $\mathrm{NH}_{2} \mathrm{COOH}-\mathrm{Y}$ & $-121.49 \pm 12.85$ & & $-5.25 \pm 0.16$ & $2.23 \pm 0.34$ & $-1.12 \pm 0.78$ \\
\hline $\mathrm{NO}_{2} \mathrm{ArCOOH}-\mathrm{Y}$ & $-115.82 \pm 15.13$ & $\square$ & $-4.65 \pm 0.15$ & $3.05 \pm 0.42$ & $-1.07 \pm 0.63$ \\
\hline
\end{tabular}


a)

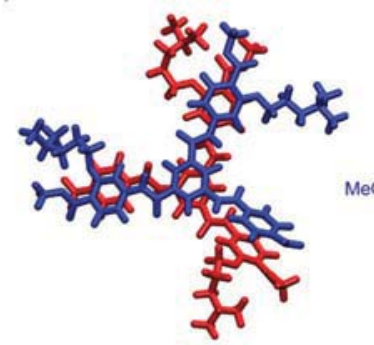

c)

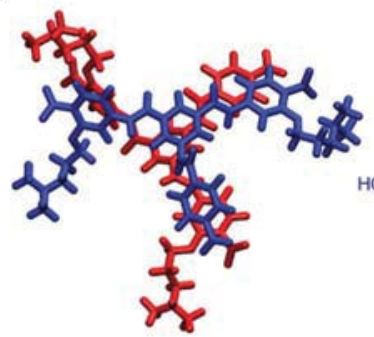

e)

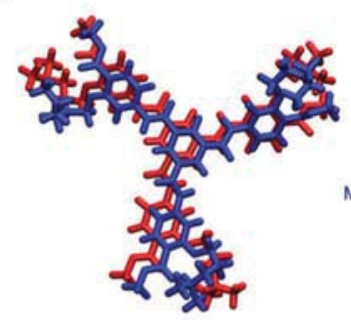

HоOC

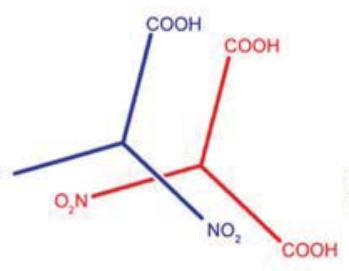

b)

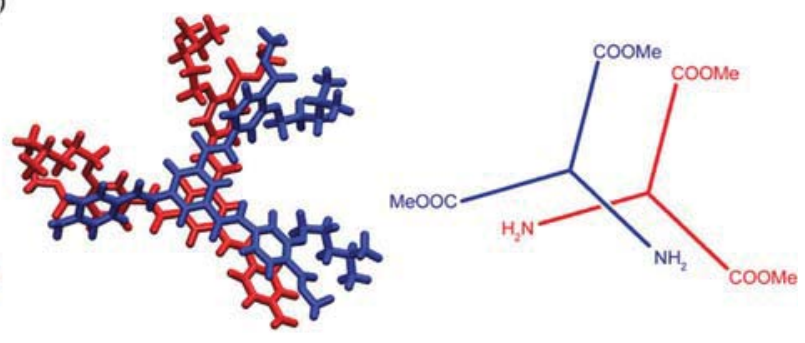

d)
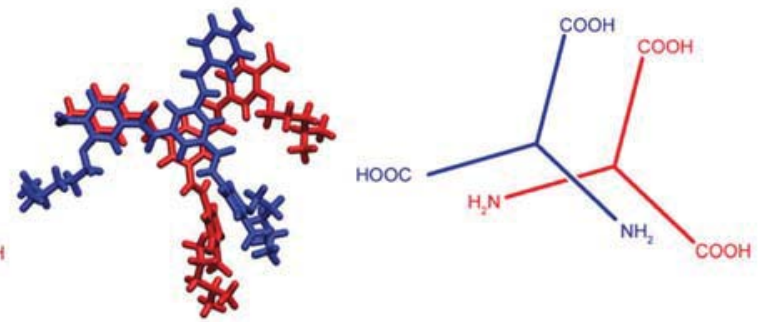

f)

g)
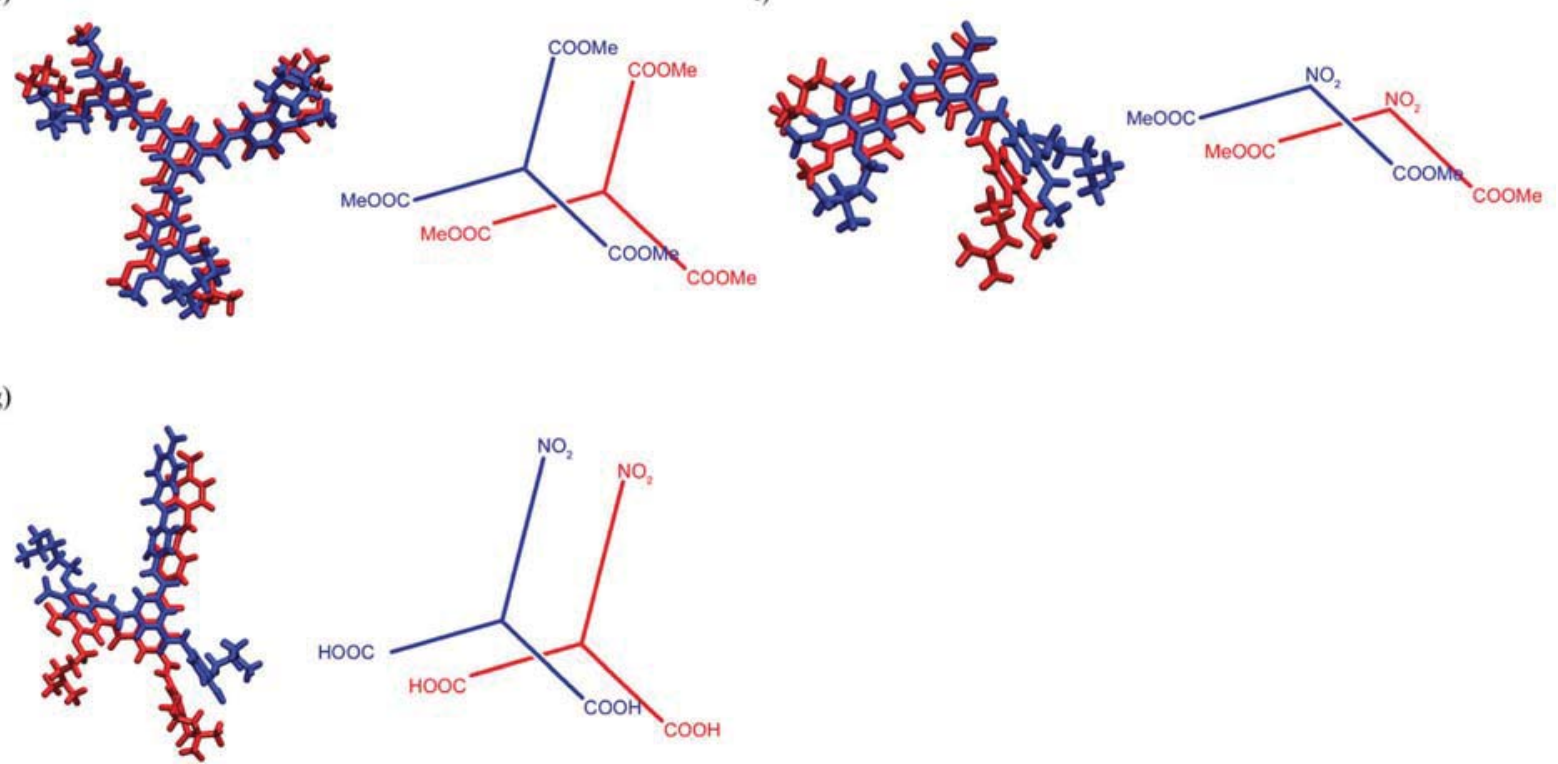

Fig. 6 Representative dimer conformation and cartoon showing the orientation of functional groups for $\mathrm{NO}_{2} \mathrm{COOMe}-\mathrm{Y}(\mathrm{a}), \mathrm{NH}_{2} \mathrm{COOMe}-\mathrm{Y}$ (b), $\mathrm{NO}_{2} \mathrm{COOH}-\mathrm{Y}(\mathrm{c}), \mathrm{NH}_{2} \mathrm{COOH}-\mathrm{Y}(\mathrm{d})$, sym(COOMe) ${ }_{3}-\mathbf{Y}(\mathrm{e})$, short $\mathrm{NO}_{2} \mathrm{COOMe}-\mathrm{Y}(\mathrm{f})$ and $\mathrm{NO}_{2} \mathrm{ArCOOH}-\mathrm{Y}(\mathrm{g})$. The methyl ester carrying tetramers (a and b) gain their dimerization energy from hydrophobic contacts but experience repulsion between their protonated amine side chains. The free carboxylic acid carrying tetramers ( $c$ and d) show less energetic gain from hydrophobic contacts but also some attractive electrostatic interactions from the interaction of the protonated side chain amine and the deprotonated carboxylic acid.

and an amino group in place of a methyl ester and a tertiary amine, as shown by the value of Debye-Hückel energy, without sacrificing hydrophobic contacts. Indeed, as shown in Fig. 6, the arrangement where nitro and amino groups are not superimposed upon each other is favored, since it minimizes the repulsion not only between the tertiary amines but also between nitro and amino groups themselves.

The dimerization free energies of $\mathbf{s y m}(\mathbf{C O O M e})_{3}-\mathbf{Y}$ and $\mathrm{NO}_{2}$ COOMe-Y are very close each other. On the other hand, the amino group of $\mathbf{N H}_{2} \mathbf{C O O M e - Y}$ monomer only reduces but does not eliminate the electrostatic repulsion with protonated amines, because of the positive partial atomic charge of its hydrogen atoms.

The aggregation of carboxylic acid tetramers $\mathrm{NO}_{2} \mathbf{C O O H}-\mathbf{Y}$ and $\mathbf{N H}_{2} \mathbf{C O O H}-\mathbf{Y}$ is caused by the synergy of hydrophobic effects and electrostatic interactions that arise from the positively charged (protonated) side chain amine and the negatively charged carboxylate, which lead to a negative and thus favorable DebyeHückel energy. Also in this case, the most probable conformation is the arrangement where nitro and amino groups are not superimposed. The stacking between rings is weaker than the methyl ester analogues as shown by the lower value of hydrophobic 
contacts; the destabilization is due to repulsion of neighboring charged groups (vide infra).

In principle, $\mathrm{NO}_{2} \mathrm{COOMe}-\mathrm{Y}, \mathrm{NH}_{2} \mathrm{COOMe}-\mathrm{Y}, \mathrm{NO}_{2} \mathrm{COOH}-\mathrm{Y}$ and $\mathbf{N H}_{2} \mathbf{C O O H}-\mathbf{Y}$ seem to offer a good balance between hydrophobic effects and electrostatic interactions, which allows building macromolecular chains and thus an interconnected network.

Short $\mathrm{NO}_{2} \mathrm{COOMe}-Y$ monomer exhibits the most unfavorable dimerization free energy. The minimum energy arrangement, shown in Fig. 6, allows on the one side maximizing hydrophobic effects with ring stacking but, on the other side, forces the superimposition of the tertiary amines and the nitro group. As a consequence, hydrophobic contacts are not able to adequately balance the unavoidable repulsion, hindering the aggregation and the attainment of large supramolecular chains.

$\mathbf{N O}_{2} \mathrm{ArCOOH}-\mathbf{Y}$ compound shows a slightly more favorable dimerization free energy, still penalized by the forced superimposition of the nitro group and by the less effective stabilization due to the neighboring charged groups, as discussed for carboxylic acid tetramers.

The observed trends can be explained also taking into account entropic contributions, since it can be assumed that tetramers experience a reduced loss of entropy with respect to the other compounds. Indeed, there are multiple accessible minimum energy configurations for the tetramers dimers in terms of stacking because of the molecular symmetry. This effect is maximum for $\mathbf{S y m}(\mathbf{C O O M e})_{3}$-Y where every arrangement that optimizes the stacking leads to a stable configuration. Such reduced entropy loss can counterbalance the higher electrostatic repulsion, thus leading to a favorable dimerization free energy value. The entropy loss is higher for the remaining tetramers because there are less accessible minimum energy configurations; indeed, some fully-stacked arrangements (that is, the ones where amino or nitro groups are superimposed) do not correspond to the minimum energy ones.

On the other side, short $\mathrm{NO}_{2} \mathrm{COOMe}-\mathrm{Y}$ and $\mathbf{N O}_{2} \mathrm{ArCOOH}-\mathrm{Y}$ dimers have a more "stiff" structure, since there is only one molecular arrangement that optimizes $\pi-\pi$ stacking and this can maximize the entropic penalty for the binding, strongly affecting dimerization free energies.

Several factors can contribute to the stabilization or destabilization of stacking from an enthalpic point of view, as shown in Fig. 7a-d for tetramers (results are valid also for the other investigated monomers). Along with the dimer structures, Fig. 7 also shows the electrostatic potential map, expressed in $k_{\mathrm{B}} T e^{-1}$ units (where $k_{\mathrm{B}}$ is the Boltzmann constant, $T$ the a)

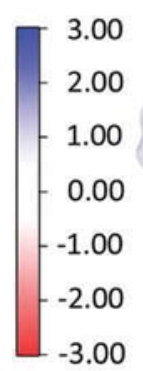

b)

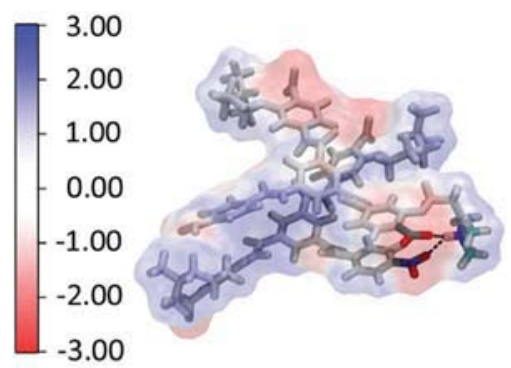

c)

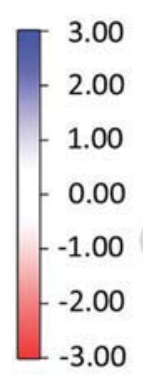

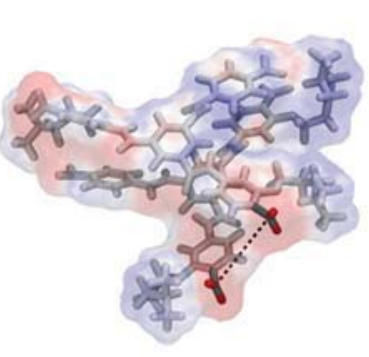

d)

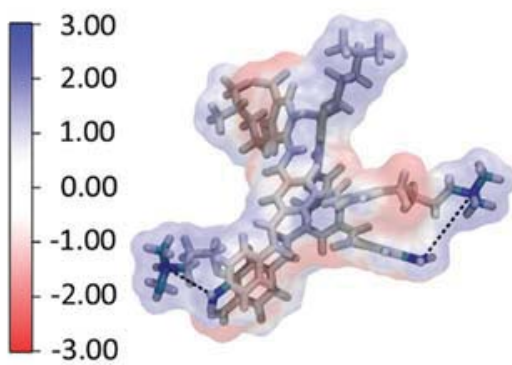

Fig. 7 (a) In the dimer of $\mathrm{NO}_{2} \mathrm{COOH}-\mathbf{Y}$ tetramers repulsion between two neighbouring negatively charged carboxylates (dashed line) can be observed. A nitro group/carboxylate repulsion observed in dimers of $\mathrm{NO}_{2} \mathrm{COOH}-\mathbf{Y}$ tetramers (b) can be partially compensated by an additional attractive interaction between the positively charged (protonated) side chain amine and the nitro/carboxylate group (dashed lines in b). (c) In dimers of $\mathrm{NO}_{2} \mathrm{COOMe-Y}$ tetramers the repulsion of neighbouring methyl esters can be partially compensated by an additional attractive interaction between the positively charged (protonated) side chain amine and the ester group (dashed line). (d) In dimers of $\mathrm{NH}_{\mathbf{2}} \mathrm{COOH}-\mathbf{Y}$ tetramers the repulsion between the positively charged (protonated) amine side chain and the positively charged protons of the aromatic amino group hinders the formation of a stable stack. 
absolute temperature and $e$ the charge of the electron) and obtained by means of the Adaptive Poisson Boltzmann Solver (APBS). In particular, blue colored areas are representative of a positive electrostatic potential, while red colored areas are characterized by a negative electrostatic potential. In particular, the shown arrangements as well as electrostatic potential maps are taken from representative snapshots from the simulation of the minimum energy dimer structure.

The stacking can be disturbed by the electrostatic repulsion between deprotonated carboxylic acids (Fig. 7a) or by the formation of a hydrogen bond between negatively charged carboxylates and the protonated tertiary amine located on the flexible short aliphatic chain (Fig. 7b).

According to our model studies, the tetramers carrying amino groups lead to a less ordered stacking than the ones carrying nitro groups. The positively charged tertiary amine on the flexible aliphatic side chain can move between the nitro group and a neighboring methyl ester/carboxylate. This reduces the electrostatic repulsion between the partially negatively charged oxygen atoms of the ester/carboxylate and hence improves stacking stability (Fig. 7c). On the other hand, the repulsion between the protonated tertiary amine and the amino group (whose hydrogen atoms have a positive partial atomic charge) can hinder the formation of a stable stack.

While the analysis of the dimer formation shed light on the fundamental driving forces behind the interactions, we focused the experimental investigation on $\mathbf{N O}_{2} \mathbf{C O O M e}-\mathbf{Y}, \mathbf{N H}_{2} \mathbf{C O O M e}-\mathbf{Y}$, $\mathrm{NO}_{2}$ COOMe-Y and $\mathbf{N H}_{2}$ COOMe-Y compounds in parallel with experimental activity, in order to better understand the impact of the composition of these very similar compounds.

The assembly of larger aggregates was firstly studied by placing 30 tetramers of each considered compound in a box filled with explicit TIP3P water molecules along with sodium and chloride ions to account for the presence of the added salt. $400 \mathrm{~ns}$ molecular dynamics simulations in NPT ensemble at $300 \mathrm{~K}$ and $1 \mathrm{~atm}$ were carried out. In all cases, the monomers experienced a spontaneous self-assembly into aggregates, whose structure is consistent with the WTMD results. The net effect, for all considered compounds, is a reduction of Solvent Accessible Surface Area (SASA), which is representative of the contribution of hydrophobic effects in the aggregation process. SASA per monomer as a function of simulation time is shown in Fig. 8 for $\mathbf{N O}_{2} \mathbf{C O O M e - Y ~ ( F i g . ~ 8 a ) ~ a n d ~} \mathbf{N O}_{2} \mathbf{C O O H}-\mathbf{Y}$ (Fig. 8b), where SASA values obtained from the simulation of a single monomer unit are included as reference. In agreement with our model we found that $\mathbf{N O}_{2} \mathbf{C O O M e}-\mathrm{Y}$ and $\mathbf{N H}_{2} \mathrm{COOMe}-\mathrm{Y}$ formed ordered aggregates while $\mathbf{N O}_{2} \mathbf{C O O H}-\mathbf{Y}$ and $\mathbf{N H}_{2} \mathbf{C O O H}-\mathbf{Y}$ arranged in one single disordered aggregate.

A more comprehensive analysis has been performed by adopting the "infinite fiber" model, successfully employed in the literature for the investigation of supramolecular chains of synthetic monomers and peptides, as recently reviewed. ${ }^{31}$ 54 tetramer units of each compound were placed in a pre-stacked conformation (according to the insights gained through the previous simulations) along the $z$ axis and solvated with explicit TIP3P water molecules along the $x$ and $y$ axes. This main element is replicated through periodic boundary conditions, allowing us to simulate the bulk of a supramolecular chain (Fig. 8c). $600 \mathrm{~ns}$ molecular dynamics simulations were carried out for $\mathbf{N O}_{2} \mathbf{C O O M e}-\mathbf{Y}$ and $\mathbf{N H}_{2} \mathbf{C O O M e}-\mathbf{Y}$, while $800 \mathrm{~ns}$ were needed to reach an equilibrated arrangement for $\mathbf{N O}_{2} \mathbf{C O O H}-\mathbf{Y}$ and $\mathbf{N H}_{2} \mathbf{C O O H}-\mathbf{Y}$ (details are reported in ESI $\dagger$ section). The last $100 \mathrm{~ns}$ of each trajectory was used for post-processing.

Energetic analyses were performed by evaluating the energy gain for a monomer to stay assembled rather than free in solution, adopting the validated approach from Garzoni and coworkers ${ }^{32}$ summarized in ESI. $\dagger$ In this framework, we focused our attention on long-range van der Waals $\left(\Delta E_{\mathrm{vdW}}\right)$ and electrostatic $\left(\Delta E_{\text {ele }}\right)$ solute-solute interactions as well as solvation free energy $\Delta G_{\text {sol }}$; the results are summarized in Table 2 .

For the methyl ester tetramers $\mathbf{N O}_{2} \mathbf{C O O M e}-\mathbf{Y}$ and $\mathbf{N H}_{2} \mathbf{C O O M e}-\mathbf{Y}$, model results show the presence of an unfavorable electrostatic repulsion (positive $\Delta E_{\mathrm{el}}$ ) given by the protonated tertiary amines, consistently with WTMD outcomes in terms of Debye-Hückel energy. This effect is almost counterbalanced by the gain in solvation free energy: stacking of neighboring tetramers leads to a reduction of the solvent exposed hydrophobic molecular surface with only the hydrophilic charged moieties accessible to the solvent.

On the other hand, the free carboxylic acid containing tetramers $\mathbf{N O}_{2} \mathbf{C O O H}-\mathbf{Y}$ and $\mathbf{N H}_{2} \mathbf{C O O H}-\mathbf{Y}$ both exhibit favorable electrostatic interactions (negative $\Delta E_{\mathrm{el}}$ ) within a stacked arrangement because of the presence of opposite charges, again consistently with WTMD results. This however overcompensated by a decrease in solvation free energy due to the fact that, in a supramolecular stack, oppositely charged groups are interacting with each other and are less exposed to the solvent. As all tetramers exhibit favorable van der Waals interactions (negative $\Delta E_{\mathrm{vdw}}$ ), whose trend is roughly similar to the hydrophobic contacts one highlighted by WTMD simulations, although the values are very close each other and essentially comparable. For the sake of completeness, $\Delta E_{\mathrm{vdw}}$ values account for the overall hydrophobic effects that involve not only aromatic ring but also e.g. the flexible aliphatic harms. To make an analogy with WTMD results, the role of hydrophobic contacts can be here better quantified by computing the stacking order through radial distribution function $g(r)$, which was calculated for the centers of mass of aromatic rings. $g(r)$ represents the probability that one aromatic ring finds a stacked aromatic ring from another tetramer at a certain distance $(r)$. The higher the value of $g(r)$, the more stable and ordered the stacking.

According to these simulations, a maximum of order and the highest tendency to form a supramolecular stack might be achievable for tetramers carrying non-ionic water soluble side chains attached via functional groups of low polarity. Synthetic efforts towards such tetramers are currently under way.

Fig. 8d shows the plot of $g(r)$ averaged over all tetramers within the fiber-like stack. Multiple peaks are present in the plot of $g(r)$, since they are related to the closest stacked neighbor, the second neighbor and so on. The observed trend of the stacking order is consistent with the WTMD results in terms of hydrophobic contacts, where $\mathrm{NO}_{2} \mathbf{C O O M e - Y}$ supramolecular chains are the most stable and persistent, while $\mathbf{N H}_{2} \mathbf{C O O H}-\mathbf{Y}$ chains show the lowest level of stacking order. 
a)

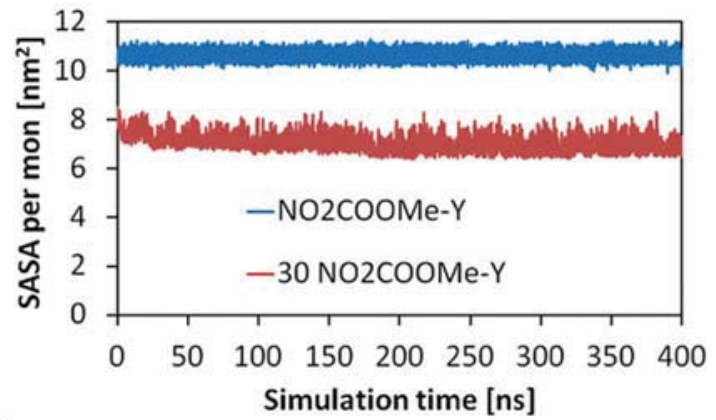

c)

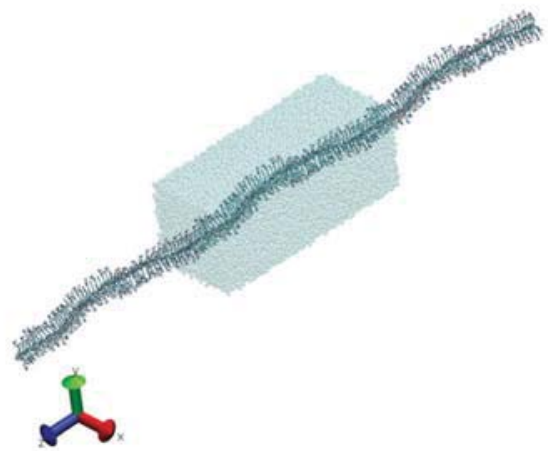

e)

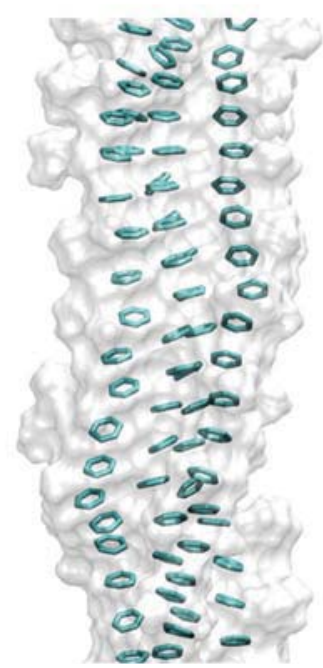

b)

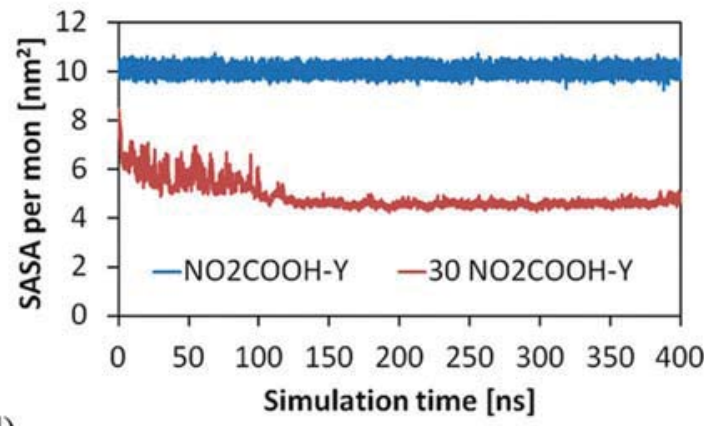

d)

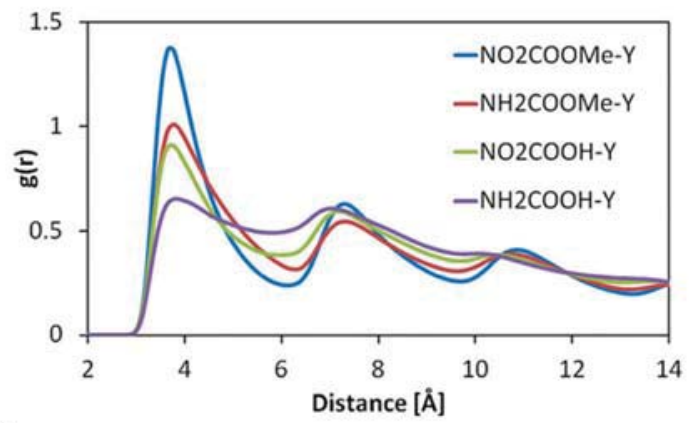

f)

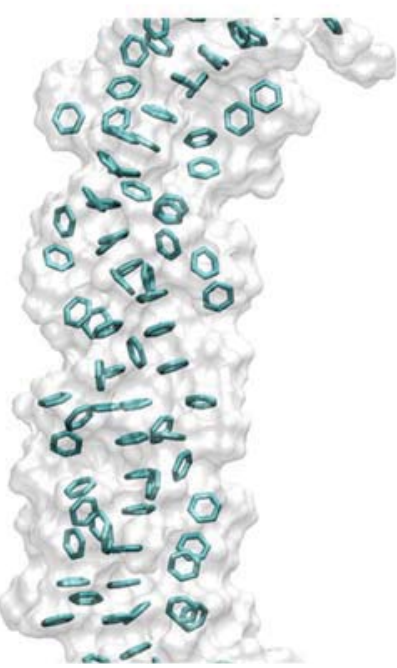

Fig. 8 Solvent accessible surface area per monomer unit for $\mathrm{NO}_{2} \mathrm{COOMe}-\mathrm{Y}(\mathrm{a})$ and $\mathrm{NO}_{2} \mathrm{COOH}-\mathrm{Y}$ (b) (red curves). SASA of single monomer in solution is shown as reference (blue curves). (c) Example of an "infinite fiber model". Simulation box is represented as a supramolecular polymer chain and solvent (transparent blue beads); the supramolecular stack is repeated twice along the $z$ axis. (d) Radial distribution function for stacked rings. (e) Equilibrated structure of $\mathrm{NO}_{2} \mathrm{COOMe}-\mathrm{Y}$ and $\mathrm{NH}_{2} \mathbf{C O O H}-\mathbf{Y}(\mathrm{f})$ supramolecular chains. Aromatic rings are explicitly shown in green, while the stack is represented as a transparent white surface.

Table 2 Energy gain for a tetramer unit to stay assembled in a 54 units supramolecular chain rather than free in solution: contributions from van der Waals interactions $\left(\Delta E_{\mathrm{vdw}}\right)$, electrostatic interactions $\left(\Delta E_{\mathrm{el}}\right)$ and solvation free energy $\left(\Delta G_{\text {solv }}\right)$

\begin{tabular}{|c|c|c|c|c|}
\hline Compound & $\Delta E_{\mathrm{vdW}}$ per unit $\left[\mathrm{kcal} \mathrm{mol}^{-1}\right]$ & $\Delta E_{\text {el }}$ per unit $\left[\mathrm{kcal} \mathrm{mol}^{-1}\right]$ & $\Delta G_{\text {solv }}$ per unit $\left[\mathrm{kcal} \mathrm{mol}^{-1}\right]$ & $\Delta E_{\mathrm{el}}+\Delta G_{\text {solv }}$ per unit $\left[\mathrm{kcal} \mathrm{mol}^{-1}\right]$ \\
\hline $\mathrm{NO}_{2} \mathrm{COOMe-Y}$ & $-41.18 \pm 2.59$ & $866.64 \pm 11.91$ & $-849.78 \pm 10.40$ & $16.86 \pm 3.31$ \\
\hline $\mathrm{NH}_{2}$ COOMe-Y & $-38.71 \pm 2.59$ & $867.80 \pm 12.06$ & $-851.66 \pm 10.71$ & $16.13 \pm 3.23$ \\
\hline $\mathrm{NO}_{2} \mathrm{COOH}-\mathrm{Y}$ & $-38.05 \pm 2.25$ & $-32.02 \pm 14.96$ & $45.70 \pm 13.73$ & $13.68 \pm 3.01$ \\
\hline $\mathrm{NH}_{2} \mathrm{COOH}-\mathrm{Y}$ & $-39.40 \pm 2.19$ & $-32.33 \pm 14.38$ & $45.30 \pm 12.89$ & $12.97 \pm 3.25$ \\
\hline
\end{tabular}


The difference between these compounds is highlighted in Fig. 8e and $\mathrm{f}$, where equilibrated supramolecular chains of $\mathbf{N O}_{2}$ COOMe-Y (Fig. 8e) and $\mathbf{N H}_{2} \mathbf{C O O H}-\mathbf{Y}$ (Fig. 8f) are reported.

For the sake of clarity, aromatic rings are explicitly represented in green, while the chain/stack is represented as a transparent white surface. While $\mathbf{N O}_{2} \mathbf{C O O M e - Y}$ aggregates exhibit an ordered stacking of aromatic rings, those of $\mathbf{N H}_{2} \mathbf{C O O H}-\mathbf{Y}$ are characterized by a more disordered arrangement.

Focusing on those compounds which lead to gel formation, the observed trend of stacking order $\left(\mathrm{NO}_{2} \mathrm{COOMe}-\mathrm{Y}>\mathbf{N O}_{2} \mathbf{C O O H}-\mathbf{Y}>\right.$ $\mathbf{N H}_{2} \mathbf{C O O H}-\mathbf{Y}$ ) is in qualitative agreement with the persistent length values obtained by fitting of SAXS profiles with a semi flexible rod model (Fig. 4) and the storage modulus values, confirming the suitability of the proposed model. On the other hand, the model does not capture the low persistence of the non-gelating $\mathbf{N H}_{2} \mathbf{C O O M e - Y}$ aggregate suggesting that a more detailed investigation is required. However, according to the SAXS results, $\mathbf{N H}_{\mathbf{2}} \mathbf{C O O M e - Y}$ might form aggregates via an entirely different mode of stacking that was not considered in our simulations. From molecular simulations, it can also be hypothesized that, while the formation of small clusters is still driven by a favorable change in free energy, the attainment of long supramolecular chains that lead to an interconnected network is hindered by the increasing importance of the repulsion between amino groups and protonated tertiary amines. The $\mathbf{N H}_{\mathbf{2}} \mathbf{C O O M e}-\mathbf{Y}$ infinite fiber simulation provided converged results, but it may constitute a metastable state, whose life time is short but still higher than the time scale of the simulation. The electrostatic repulsion can create some defects in the fiber that subsequently lead to the release of a monomer in the surrounding solution or the rupture of the supramolecular chain, thus hindering the formation of a stable network. The structural complexity of the involved monomer and the characteristic time scale of molecular dynamics simulations do not allow investigating this process in detail at atomic scale. As shown in literature, a convenient way can be constituted by coarse-grained (CG) models accurately parameterized from atomistic simulations ${ }^{33}$ that allow accessing higher time and length scales than standard MD simulations and potentially observing the attainment of defects. In addition, the use of infrequent metadynamics coupled with CG models proved to be a suitable choice to compute the characteristic time scale of monomer exchange between the assembly and the surrounding solution. ${ }^{34}$ At this stage, the development of CG models for the investigated oligomers and the study of their assembly at CG scale is beyond the purpose of this paper but it is considered for further investigations.

\section{Conclusions}

In this paper, we presented the synthesis and design of a set of amphiphilic aramid oligomers capable to serve as LMWG for water. By varying the functional groups present on the constituting oligomers the interplay of different non-covalent interactions, such as aromatic stacking and ionic interactions, is strongly influenced. Gel formation was investigated via rheology measurements regarding the dependence on temperature and $\mathrm{pH}$ values. Rheology and electron microscopic analysis of the aqueous gels revealed that smallest structural changes on the oligomeric building blocks resulted in large changes in the observed self-assembled structures and the ability to form gels in water.

In order to be able to understand the self-assembly process on a molecular level and thus be able to rationally modify gelators like the ones investigated here in the future we followed the selfassembly process via molecular dynamics simulations. To gain further insight into the arrangement of oligomers within the stack and the influence of functional groups on the orientation and strength of intermolecular interactions between oligomers, we first of all studied the formation of dimers by means of welltempered metadynamics simulations. MD simulations revealed that all compounds investigated self-assembled in water to some extent, but the key role in the aggregation process is played by stacking order rather than monomer solubility. The stacking order obtained from MD simulations agrees qualitatively with the persistence length values determined from small angle X-ray scattering of the gelators.

The present study can serve well as a model for the design of LMWG supported by molecular dynamics simulations. Our report shows that such methods can be valuable aids for understanding self-assembly processes in synthetic supramolecular chemistry.

\section{Experimental section}

\section{Preparation of hydrogels}

All potential gelators are synthesized as described in the ESI. $\dagger$ For the preparation of hydrogels, the desired weight percentage was filled in a small vial equipped with a tiny stir bar. During investigation of the gel properties it became clear that the influence of temperature plays an important, non-reversible role. For that reason, it was made sure that the preparation of gels was always carried out at constant temperatures to guarantee following experiments deliver reproducible results. This concerns above all rheology investigations. It was shown that the gels prepared at higher temperatures show lower storage moduli. This topic will be discussed later. In order to be independent from fluctuating environmental temperatures, all gels were prepared in an ice cooled water-bath, which was not allowed to exceed $10{ }^{\circ} \mathrm{C}$.

\section{UV-vis measurements}

For UV-vis experiments a UV-vis spectrophotometer V-630 (Jasco, Japan) in absorbance mode with a speed of $200 \mathrm{~nm} \mathrm{~min}^{-1}$ and an interval of $0.2 \mathrm{~nm}$ was used with a $10 \mathrm{~mm}$ matched quartz cell. Concentrations indicated in respective experiments.

\section{Small-angle X-ray scattering (SAXS)}

Hydrogel samples ( $3 \mathrm{wt} \%$ in $0.01 \mathrm{M} \mathrm{NaCl}_{\mathrm{aq}}$ ) were evaluated via SAXS. Wet gel samples were places in a quartz glass capillary tube (0.2 mm diameter, one side open, Capillary Tube Supplies Ltd, UK) and sealed vacuum-tight with conventional two-component 
epoxy glue. Spectra have been recorded using a MicroMax 003 Microfocus (Rigaku Innovative Technologies, Auburn Hills, MI USA) with a $\mathrm{Cu}$ target sealed tube source. The scattering data were collected using a Pilatus100 K detector (Dectris). SAXS measurements were conducted under vacuum at $20{ }^{\circ} \mathrm{C}$. The sample-to-detector distance was calibrated using a silver behenate standard. SAXS intensities are reported as function of scattering vector $q$ defined as $q=4 \pi \sin (\theta / 2) \lambda^{-1}$ with a photon wavelength of $\lambda=0.1524 \AA$.

\section{Microscopy}

To determine the manner of aggregation via Transmission Electron Microscopy (TEM) imaging a drop of sample solutions $\left(c=2.5 \mathrm{mg} \mathrm{mL}{ }^{-1}\right.$ in $\left.10 \mathrm{mM} \mathrm{NaCl}_{\mathrm{aq}}\right)$ were deposited onto a carbon-coated copper grid placed on a filter paper to remove excess solution. The grid was left drying overnight in a Petri dish protected from dust and imaged with a Tecnai Spirit (BioTwin lens, operated at $120 \mathrm{kV}$ LaB6 emitter) transmission electron microscope (FEI, Netherlands) equipped with a Valeta $2048 \times 2048$ wide angle detector. Atomic force microscopy (AFM) was carried out with a Nanosurf FlexAFM in tapping mode with a silicone probe tip (resonant frequency $190 \mathrm{kHz}$ and constant force of $48 \mathrm{~N} \mathrm{~m}^{-1}$ ). Images were analyzed using SPIP software. For sample preparation solutions $(2.5 \times$ $10^{-2} \mathrm{mg} \mathrm{mL}^{-1}$ in $\left.10 \times 10^{-2} \mathrm{mM} \mathrm{NaCl}_{\mathrm{aq}}\right)$ were drop-cast on mica sheets (G250-2, $100 \times 100$, Agar Scientific Ltd, Stansted, UK) and left drying overnight protected from dust. For SEM measurements freeze-dried xerogels $(3 \mathrm{wt} \%$ gels in $10 \mathrm{mM}$ $\mathrm{NaCl}_{\mathrm{aq}}$ ) have been deposited on a SEM sample holder, coated with gold and investigated with a Tescan MIRA3 LM FE (fieldemission: Schottky emitter, detector: In-Beam SE, voltage: $5 \mathrm{kV}$ ).

\section{Rheology}

In order to investigate the viscoelastic spectrum of the hydrogels under different conditions rheological experiments were carried out on an AR G2 rheometer (TA Instruments, New Castle, DE) equipped with a parallel plate set-up. After a conditioning step at $25{ }^{\circ} \mathrm{C}$, storage modulus $\left(G^{\prime}\right)$ and loss modulus $\left(G^{\prime \prime}\right)$ were recorded as a function of angular frequency in a range from $0.1 \mathrm{rad} \mathrm{s}^{-1}$ to $100 \mathrm{rad} \mathrm{s}^{-1}$ at a controlled strain of $1 \%$ (frequency sweep test). A subsequent amplitude sweep test was conducted to investigate the moduli in dependence of strain between $0.1-1000 \%$ at a fixed angular frequency of $1 \mathrm{~Hz}$.

\section{Conflicts of interest}

There are no conflicts to declare.

\section{Acknowledgements}

SD and AFMK thank the EU ITN "SASSYPOL" and the National Center for Competence in Research (NCCR) "Bio-inspired Materials" for financial support. TEM, SEM, SAXS and rheological experiment could kindly be performed at the Adolphe-Merkle-Institute, University of Fribourg (Switzerland). Furthermore, this work benefited from the use of the SasView application, ${ }^{21}$ originally developed under NSF award DMR-0520547. SasView contains code developed with funding from the European Union's Horizon 2020 research and innovation programme under the SINE2020 project, grant agreement No. 654000. The authors acknowledge the computational resources provided by ETH Zürich with Euler cluster. T. C. acknowledges Michela Castelnuovo, B. Des. for image editing and Davide Bochicchio, $\mathrm{PhD}$ for useful discussions.

\section{References}

1 L. Yang, X. Tan, Z. Wang and X. Zhang, Chem. Rev., 2015, 115, 7196-7239.

2 E. Krieg, M. M. C. Bastings, P. Besenius and B. Rybtchinski, Chem. Rev., 2016, 16, 2414-2477.

3 G. Yu, K. Jie and F. Huang, Chem. Rev., 2015, 115, 7240-7303.

4 M. R. Sambrook and S. Notman, Chem. Soc. Rev., 2013, 42, 9251-9267.

5 X. Ma and Y. Zhao, Chem. Rev., 2015, 115, 7794-7839.

6 X. Du, J. Zhou, J. Shi and B. Xu, Chem. Rev., 2015, 115, 13165-13307.

7 C. D. Jones and J. W. Steed, Chem. Soc. Rev., 2016, 45, 6546-6596.

8 J. Plante, F. Campbell, B. Malkova, C. Kilner, S. L. Warriner and A. J. Wilson, Org. Biomol. Chem., 2008, 6, 138-146.

9 N. Busschaert, S. Thompson and A. D. Hamilton, Chem. Commun., 2017, 53, 313-316.

10 I. Saraogi and A. D. Hamilton, Chem. Soc. Rev., 2009, 38, 1726-1743.

11 Y. Zhang, R. Cao, J. Shen, C. S. F. Detchou, Y. Zhong, H. Wang, S. Zou, Q. Huang, C. Lian, Q. Wang, J. Zhu and B. Gong, Org. Lett., 2018, 20, 1555-1558.

12 I. Huc, Eur. J. Org. Chem., 2004, 17-29.

13 Z. T. Li, J. L. Hou, C. Li and H. P. Yi, Chem. -Asian J., 2006, 1, 766-778.

14 D. W. Zhang, X. Zhao, J. L. Hou and Z. T. Li, Chem. Rev., 2012, 112, 5271-5316.

15 A. J. Wilson, J. Van Gestel, R. P. Sijbesma and E. W. Meijer, Chem. Commun., 2006, 4404-4406.

16 E. R. Gillies, F. Deiss, C. Staedel, J. Schmitter and I. Huc, Angew. Chem., Int. Ed., 2007, 46, 4081-4084.

17 B. Baptiste, C. Douat-Casassus, K. Laxmi-Reddy, F. Godde and I. Huc, J. Org. Chem., 2010, 75, 7175-7185.

18 X. Hu, S. J. Dawson, P. K. Mandal, X. De Hatten, B. Baptiste and I. Huc, Chem. Sci., 2017, 8, 3741-3749.

19 P. Rajdev, S. Chakraborty, M. Schmutz, P. Mesini and S. Ghosh, Langmuir, 2017, 33, 4789-4795.

20 S. R. Amrutha and M. Jayakannan, J. Phys. Chem. B, 2008, 112, 1119-1129.

21 SasView, http://www.sasview.org, accessed 3 November 2017.

22 R. G. Weiss, J. Am. Chem. Soc., 2014, 136, 7519-7530.

23 G. Ren, D. Hu, E. W. C. Cheng, M. A. Vargas-Reus, P. Reip and R. P. Allaker, Int. J. Antimicrob. Agents, 2009, 33, 587-590.

24 T. J. Moyer, J. A. Finbloom, F. Chen, D. J. Toft, V. L. Cryns and S. I. Stupp, J. Am. Chem. Soc., 2014, 136, 14746-14752. 
25 C. Ou, J. Zhang, X. Zhang, Z. Yang and M. Chen, Chem. Commun., 2013, 49, 1853-1855.

26 A. R. Hirst, I. A. Coates, T. R. Boucheteau, J. F. Miravet, B. Escuder, V. Castelletto, I. W. Hamley and D. K. Smith, J. Am. Chem. Soc., 2008, 9113-9121.

27 B. Jayaram, D. Sprous and D. L. Beveridge, J. Phys. Chem. B, 1998, 102, 9571-9576.

28 A. Onufriev, D. Bashford and D. A. Case, Proteins: Struct., Funct., Genet., 2004, 55, 383-394.

29 A. Onufriev, D. Bashford and D. A. Case, J. Phys. Chem. B, 2000, 104, 3712-3720.
30 A. Barducci, G. Bussi and M. Parrinello, Phys. Rev. Lett., 2008, 100, 1-4.

31 D. Bochicchio and G. M. Pavan, Adv. Phys.: X, 2018, 3, 1436408.

32 M. Garzoni, M. B. Baker, C. M. A. Leenders, I. K. Voets, L. Albertazzi, A. R. A. Palmans, E. W. Meijer and G. M. Pavan, J. Am. Chem. Soc., 2016, 138, 13985-13995.

33 D. Bochicchio and G. M. Pavan, ACS Nano, 2017, 11, 1000-1011.

34 D. Bochicchio, M. Salvalaglio and G. M. Pavan, Nat. Commun., 2017, 8, 147. 